\title{
Synthesis and characterization of a new type of electro-optic polymer without carbon main chains
}

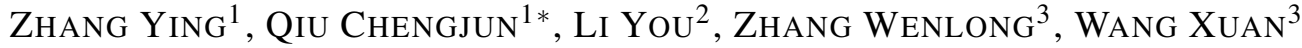 \\ ${ }^{1}$ Electronic Engineering College of Heilongjiang University, Harbin, 150080, China \\ ${ }^{2}$ Harbin Research Institute of Electrical Instrument, Harbin, 150028, China \\ ${ }^{3}$ State Key Laboratory Breeding Base of Dielectric Engineering, Harbin, 150080, China
}

\begin{abstract}
A new type of electro-optic (EO) polymer is prepared in this work. The main chain of the EO polymer is made of polyphosphazenes, and the side chain consists of carbazole based nitro azobenzene. The principle and method of preparation are given and the characteristics of this material are studied in details. The polymer with an EO coefficient of $35 \mathrm{pm} / \mathrm{V}$ has fine stability and can be easily processed. It also shows photoconductivity due to the carbazole group. This polymer thin film is obtained through performance improvement. Terahertz ( $\mathrm{THz}$ ) wave can be detected using the device, which is made of the new polymer. Owing to its fascinating properties, this new type of EO polymer has the potential to be widely applied in photorefractive materials as well as for emission and detection of $\mathrm{THz}$ radiation.
\end{abstract}

Keywords: EO polymer; polyphosphazenes; carbazole based nitro azobenzene; THz radiation

(C) Wroclaw University of Technology.

\section{Introduction}

Polyphosphazenes, based on phosphorus and nitrogen atoms, are inorganic polymeric functional materials without carbon main chains. This kind of materials is very stable against temperature, oil, radiation, solvents and so on. There are side groups of $R_{1}$ and $R_{2}$ that are connected to every phosphorus atom. Many kinds of functions can be gained with suitable R-group. Polyphosphazenes are realized by synthesis a flame resistant material, special rubber and an elastic material at low temperature. Nowadays, due to its unique functional properties, polyphosphazenes have been developed and utilized in biomedicine, liquid crystals, aerospace, power electronics, energy storage and generation, membrane science, elastomers, surface science, photoelectric functional materials, military manufacturing and biomedical science $[1,2]$. The research on polyphosphazenes development progresses rapidly all over the world, while rather slow in China. With the discovery

*E-mail: waterunderbridge@163.com of its superiority, polyphosphazene has been a research focus of most scholars [3-5].

A kind of polymer material based on polyphosphazenes and EO material with polyvinyl carbazole based nitro azobenzene as nonlinear optical (NOL) molecule is studied in this paper. The NOL molecule is connected to polyphosphazene main chain as a side group that gives rise to the polymer electro-optic properties. The principle, method of preparation and the characteristics of this material are studied in details. Comparing with the hostguest structure polymer, this kind of polymer is more stable and can be processed more easily. With an EO coefficient of $35 \mathrm{pm} / \mathrm{V}$, the polymer has fine electro-optic properties as well as perfect photoconductivity. Through the optimization of the polymer, an EO polymer film with fine film-forming property, light absorbing property and perfect EO coefficient can be gained. The $\mathrm{THz}$ wave can be well detected with the device made of the polymer discussed above. This kind of polymer material can be widely applied in photorefractive materials $[6,7]$ as well as for emission and detection of $\mathrm{THz}$ radiation [8-10]. 


\section{Experimental setup and results}

\subsection{Synthesis of the material}

The working temperature can be significantly enhanced if the main chain is not composed of carbon atoms. The main chain of polyphosphazenes is composed of phosphorus atoms and nitrogen atoms, so polyphosphazene containing carbazole grafted azo nitrobenzene is synthesized in order to improve temperature characteristics.

\section{Synthesis of N-hydroxyethyl carbazole.}

The N-hydroxylethyl carbazole is synthesized by chemical reactions with carbazole, potassium hydroxide and 2-chloroethanol, as raw materials, and dimethyl-formamide (DMF) as a solvent. The chemical reaction is given by:<smiles>OCCn1c2ccccc2c2cc(-c3ccccc3)ccc21</smiles>

\section{Synthesis of polydichlorophosphazene.}

Polydichlorophosphazene can be obtained by ring opening polymerization of hexachlorocyclotriphosphazene under a given temperature. The chemical reaction is given by:<smiles>ClP1(Cl)=NP(Cl)(Cl)=NP(Cl)(Cl)=N1</smiles>

Synthesis of polyphosphazene containing carbazole.

The polyphosphazene containing carbazole has been synthesized by nucleophilic substitution reactions with polydichlorophosphazene and $\mathrm{N}$ hydroxylethyl carbazole. The chemical reaction is given by:

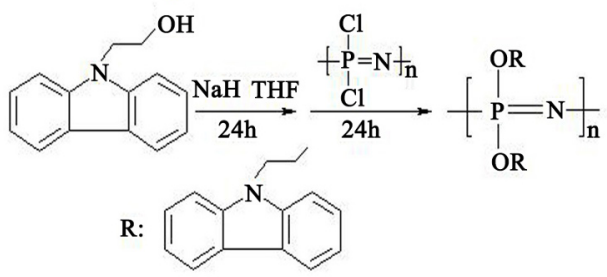

Synthesis of polyphosphazene containing carbazole grafted by azo nitrobenzene.

The polyphosphazene containing carbazole grafted by azo nitrobenzene has been synthesized by chemical reactions with polyphosphazenes containing carbazole, potassium bisulfate, P-nitroaniline and sodium nitrite, as raw materials, while heated in a microwave oven. The material has been obtained by only one step reaction, which is simple and fast. The chemical reaction is shown in the equation (4) and the effect of different microwave powers on the material yields is listed in Table 1. As one can see, the microwave power of $400 \mathrm{~W}$ and above is desirable.

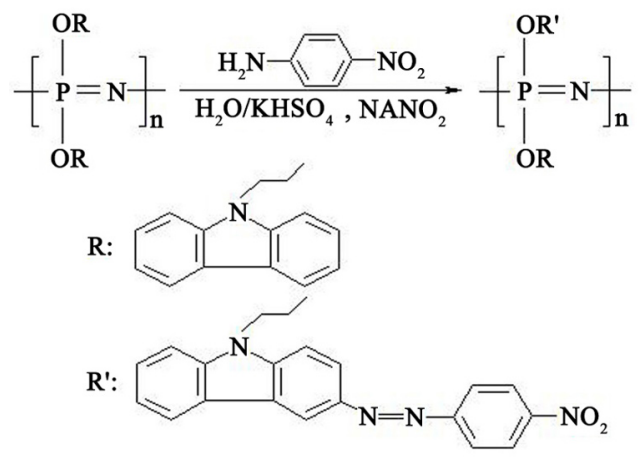

Table 1. The effect of different microwave powers on material yields.

\begin{tabular}{c|c|c|c|c|c|c}
\hline microwave power [W] & 100 & 200 & 300 & 400 & 500 & 600 \\
\hline productivity [wt.\%] & 48.6 & 55.2 & 59.6 & 63.5 & 61.4 & 62.4 \\
\hline
\end{tabular}

\subsection{Structure characterization}

UV-visible absorption spectrum test has been made to study the polyphosphazene containing carbazole grafted by azo nitrobenzene with an UV757CRT photometer. The concentration of the tetrahydrofuran solvent (THF) is $104 \mathrm{~mol} / \mathrm{L}$. The result is shown in Fig. 1.

There is no absorption in the visible light region. The peaks obtained at $330 \mathrm{~nm}$ and $343 \mathrm{~nm}$ are the absorption peaks of carbazole groups, which are the result of the $\pi-\pi^{*}$ transition. The absorption at $409 \mathrm{~nm}$ is the reason that the extended chromophores conjugated structure is induced by power 


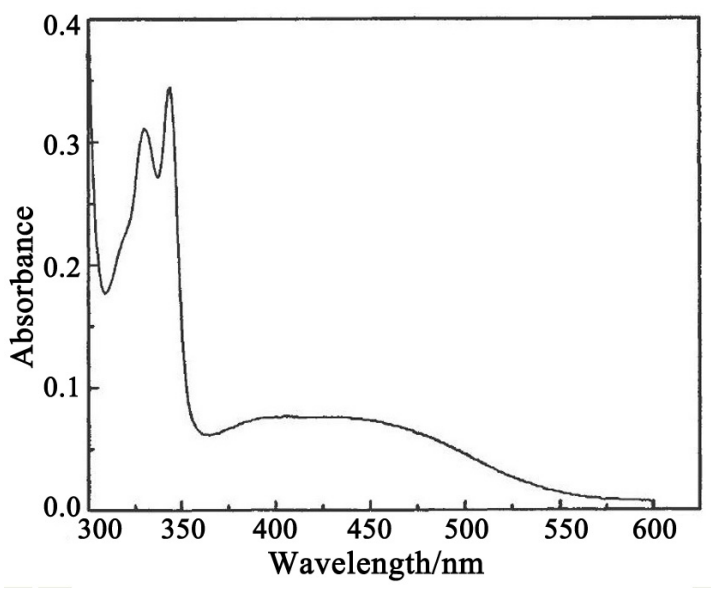

Fig. 1. The UV-visible absorption spectrum of polyphosphazenes containing carbazole grafted by azo nitrobenzene.

groups, which is the result of the $\mathrm{n}-\pi *$ transition in azo group.

Infrared (IR) absorption spectrum test has been made to the polyphosphazenes containing carbazole grafted by azo nitrobenzene with an EQUINOX55 IR/Raman spectrometer based on $\mathrm{KBr}$ discs and with the resolution of $8 \mathrm{~cm}^{-1}$. The result is shown in Fig. 2.

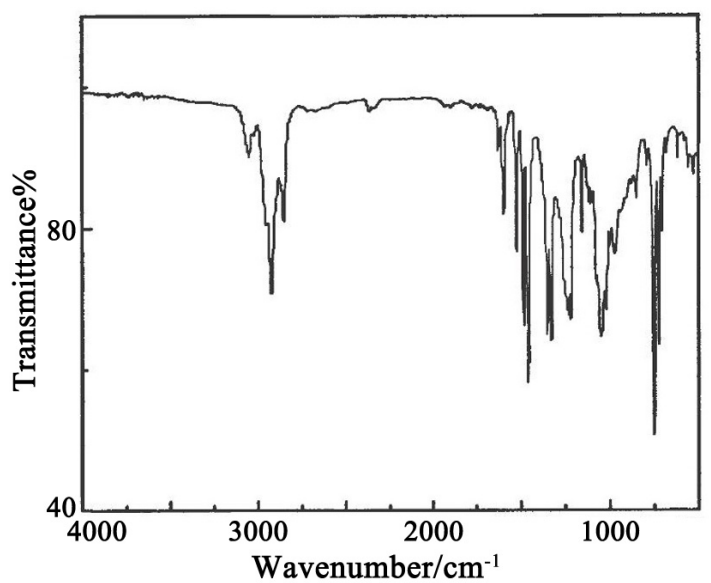

Fig. 2. The IR spectrum of polyphosphazene containing carbazole grafted by azo nitrobenzene.

The characteristic absorption peaks of the carbazole appear at $3050 \mathrm{~cm}^{-1}, 1600 \mathrm{~cm}^{-1}$, $1484 \mathrm{~cm}^{-1}, 1450 \mathrm{~cm}^{-1}$. The stretch absorption peak of the $\mathrm{C}-\mathrm{H}$ on fatty chain is obtained at
$2930 \mathrm{~cm}^{-1}$. The characteristic absorption peaks of the $\mathrm{P}-\mathrm{O}-\mathrm{C}$ appear at $1050 \mathrm{~cm}^{-1}$ and $926 \mathrm{~cm}^{-1}$, while the peaks of the $\mathrm{P}=\mathrm{N}$ appear at $1320 \mathrm{~cm}^{-1}$, $1240 \mathrm{~cm}^{-1}$. The stretch absorption peak of P-N is obtained at $750 \mathrm{~cm}^{-1}$ and $723 \mathrm{~cm}^{-1}$. These absorption peaks observed indicate that the carbazole is grafted on the polydichlorophosphazene successfully.

\subsection{Property tests}

\section{EO properties.}

A sample having fine structure whose thickness is more than $200 \mu \mathrm{m}$ can be obtained by the spin coating technology. One side of the sample is coated with indium tin oxide (ITO) electrode and polarized through corona poling. The other side of the sample is coated with Al electrode for the measurement of EO coefficient and photoconductivity. The picture of the sample is shown in Fig. 3. This kind of polymer is more stable than the conventional host-guest structure polymer. For the conventional host-guest structure polymer, the EO molecule is separated after a period of time, which leads to reduction of the EO coefficient and transparency of the sample. Taking the reduction of $10 \%$ as a parameter to characterize stability, the time of stabilization of a conventional host-guest structure polymer is typically around two weeks $[11,12]$. Stability of this new polymer is improved drastically as the transparency and EO coefficient remain unchanged after one-year time.

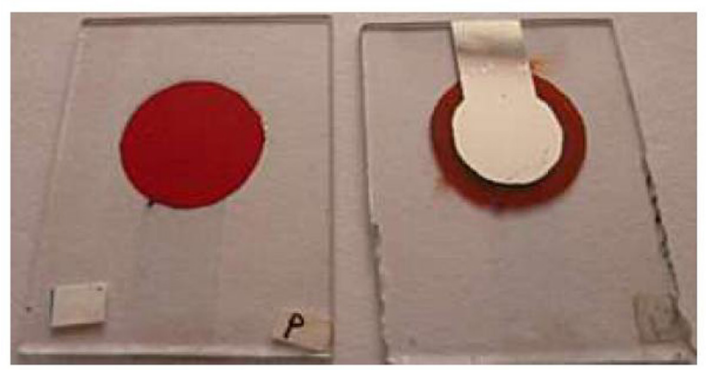

Fig. 3. The photo of the sample.

The EO coefficient is tested with a simple reflection method [13]. The principle of the test is shown in Fig. 4. In an orthogonal polarization system, the refractive index of the EO polymer 
will be altered because of the modulation of the electric field. Then the EO coefficient of the sample can be obtained by measuring the phase change that is caused by light intensity change.

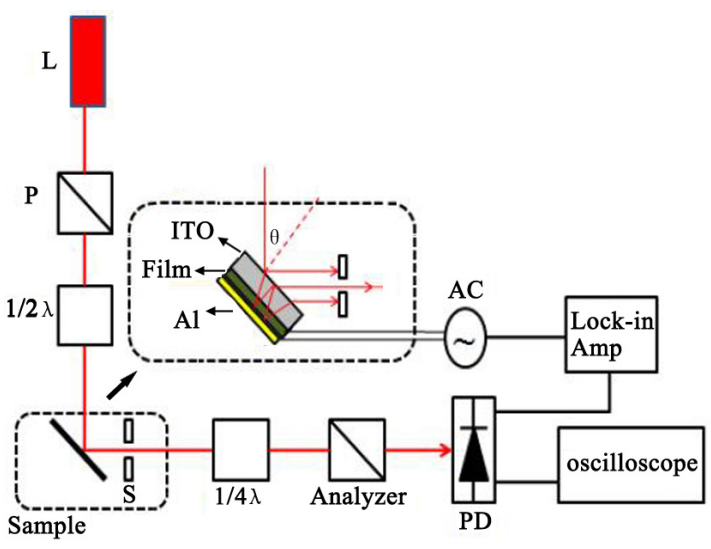

Fig. 4. Principle of a simple reflection method.

The orthogonal polarization system is composed of two polarizes, and L stands for the laser source. A He-Ne laser with the wavelength of $632.8 \mathrm{~nm}$ and the power of $2 \mathrm{~mW}$ has been used in this experiment. The angle between the light passing through the analyzer $\mathrm{P}$ and the sample is 45 degree. The half-wave plate is used to control the polarization plane of the incident light. A part of the light is reflected when the incident light reaches the interface of the air and the ITO. The other part of the light gets into the polymer and is reflected by the Al layer. Finally, a part of the light gets out of the ITO, but the other one stays in the polymer where the reflection happens for several times. In order to prevent the reflection, a diaphragm is used between the half-wave plate and the sample. The change of the light intensity caused by alternate current $(\mathrm{AC})$ is measured by a lock-in amplifier. An oscilloscope is used to measure the voltage controlled by the half-wave plate.

The wavelength of the laser is $632.8 \mathrm{~nm}$, while the voltage peak is $16.5 \mathrm{~V}$. The EO coefficient $\gamma_{33}=35 \mathrm{pm} / \mathrm{V}$ when the grafting rate is $18.8 \mathrm{wt} . \%$.

\section{Photoconductivity.}

The polymer shows photoconductivity because of the carbazole group. The principle of testing photoconductivity is shown in Fig. 5.

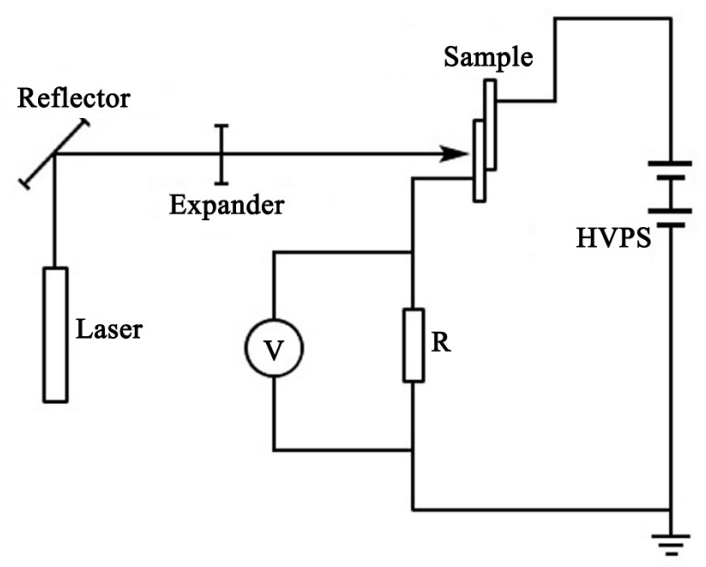

Fig. 5. Principle of testing photoconductivity.

The wavelength of the laser for photoconductivity testing is $532 \mathrm{~nm}$. The result of the test is shown in Fig. 6 under different luminous powers when the grafting rate is $18.8 \mathrm{wt} . \%$. At the same luminous power, the photoconductivity increases as the field intensity increases, which indicates that the carrier mobility is improved. Also the electrical conductivity is improved at higher electric field intensity. At the same electric field intensity, the photoconductivity increases as the luminous power intensity becomes larger, which indicates that the number of carriers increases because of the increased light intensity.

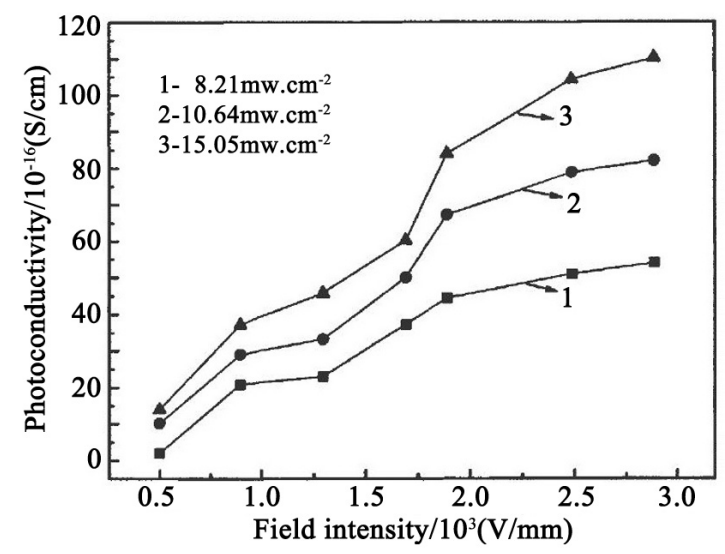

Fig. 6. Characteristic curves of photoconductivity.

\section{THz characteristics.}

Based on the sample of polyphosphazene containing carbazole grafted by azo nitrobenzene, double 
electro-optic molecules polymer can be obtained through small EO molecule physical doping. Ethyl 4-dimethylaminobenzoate (EDB) has been chosen as a small EO molecule for the experiment to improve the EO coefficient. The THz wave detector has been prepared with the doping ratio of $7.5 \mathrm{wt} . \%$ and the polarization temperature of $75^{\circ} \mathrm{C}$. The time domain waveform of $\mathrm{THz}$ wave is shown in Fig. 7.

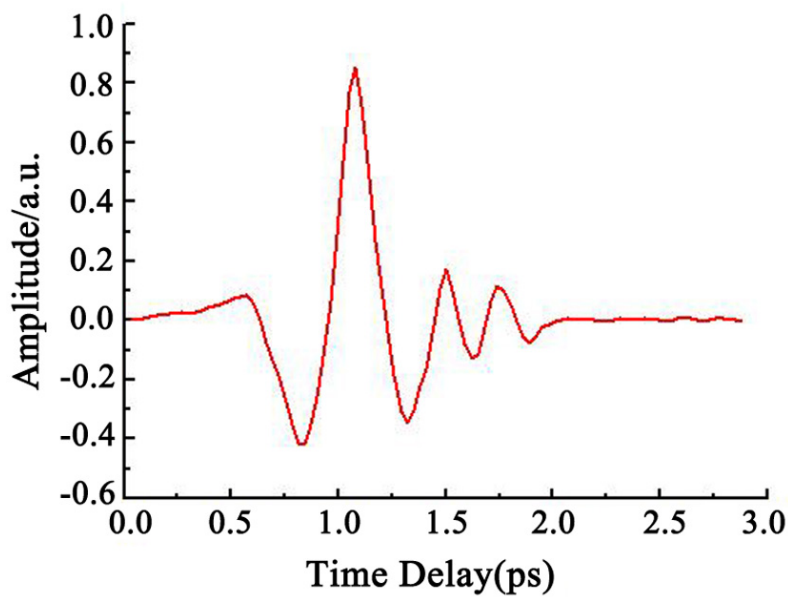

Fig. 7. Time domain waveform of $\mathrm{THz}$ wave.

The full width at half maximum (FWHM) of the $\mathrm{THz}$ wave is about $160 \mathrm{fs}$ and the duration is 1.6 ps. The frequency spectrum is shown in Fig. 8 . The FWHM of the $\mathrm{THz}$ frequency spectrum is $1.53 \mathrm{THz}$, the cut-off frequency is about $2.8 \mathrm{THz}$ and the peak frequency is $1.3 \mathrm{THz}$. These results indicate that the $\mathrm{THz}$ wave can be detected by the polymer.

\section{Conclusions}

Polyphosphazenes containing carbazole grafted by azo nitrobenzene have been prepared by a microwave heating method, and the obtained material has excellent stability and processing characteristics. The experiments indicate that when the grafting rate is $18.8 \mathrm{wt} . \%$, the EO coefficient is $35 \mathrm{pm} / \mathrm{V}$. The polymer has good EO properties as well as photoconductivity. Under certain luminous power intensity, the photoconductivity increases as the electric field intensity becomes larger. For a given

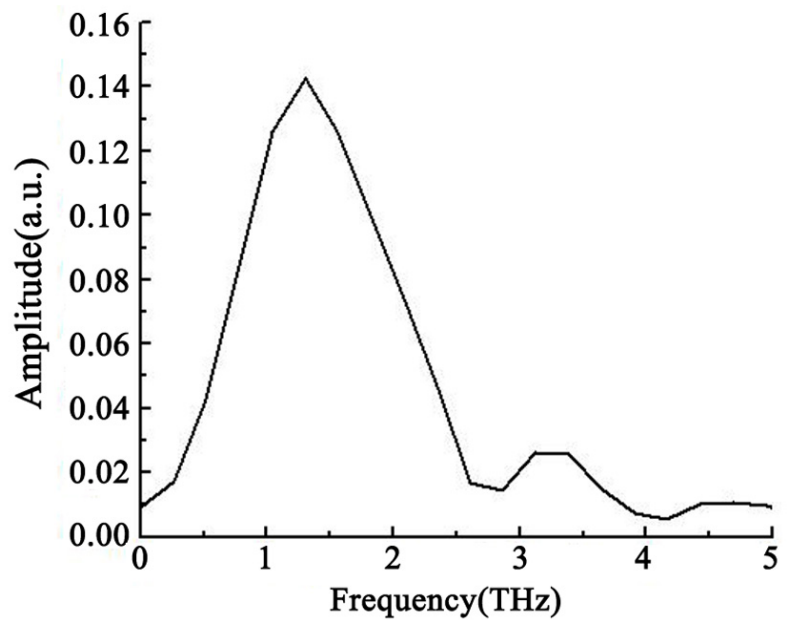

Fig. 8. Frequency spectrum of $\mathrm{THz}$ wave.

electric field intensity, the photoconductivity of the material increases as the light intensity becomes larger. $\mathrm{THz}$ wave can be detected clearly by the polymer based on the new EO polymer. This new type of EO polymer without carbon main chains is an excellent photorefractive material, which will open new opportunities for emission and detection of $\mathrm{THz}$ radiations.

\section{Acknowledgements}

The work is supported by the National Natural Foundation of China (60871073) and the Research Foundation of Education Bureau of Heilongjiang Province (11551076, 12511078).

\section{References}

[1] Harry R., Appl. Organomet. Chem., 27 (2013), 620.

[2] Zhang H., Li A., Shen P., Mater. Rev., 24 (2010), 133.

[3] Zhou L., Su Y., Sci. Technol. Chem. Ind., 20 (2012), 68.

[4] Bao D., LiU J., China Plast., 26 (2012), 16.

[5] Harry R., Polym. Prepr. (Am. Chem. Soc., Div. Polym. Chem.), 41 (2000), 553.

[6] Henke H., Wilfert S., Iturmendi A., J. Polym. Sci. Pol. Chem., 51 (2013), 4467.

[7] Muhsin E., Jayan T., Savas T., Appl. Phys. Lett., 85 (2004), 1095.

[8] Zhang W., Zhao C., J. Funct. Mater., 43 (2012), 3386.

[9] Shan J., Weling A.S., Knoesel E., Bartels L., Bonn M., Nahata A., Reider G.A., Heinz T.F., Opt. Lett., 25 (2000), 426.

[10] Harry R., Curr. Opin. Solid St. M., 10 (2006), 231.

[11] Sinyukov A.M., Hayden L.M., Opt. Lett., 27 (2002), 55. 
[12] Yu F., Spring A.M., Li L., QiU F., Yamamoto K., Maeda D., Ozawa M., Odoi K., Yokoyama S., $J$. Polym. Sci. Pol. Chem., 51 (2013), 1278.

[13] Michelotti F., Toussaere E., Levenson R., Received 2014-04-07 LiAng J., Zyss J., Appl. Phys. Lett., 67 (1995), 2765. 